\title{
Consenso Global sobre la Responsabilidad Social de las Facultades de Medicina ${ }^{1}$
}

\author{
Charles Boelen, Robert Woollard (copresidentes)
}

A comienzos del siglo $\mathrm{xx}$ las facultades de medicina se enfrentaban al reto sin precedentes de llegar a ser más científicas y efectivas en la formación de médicos, según quedó reflejado en el informe Flexner de 1910. El siglo XXI confronta las facultades de medicina con un conjunto de retos distintos: la mejora de la calidad, la equidad, la relevancia y la efectividad en la prestación de los servicios asistenciales, reducción de los desajustes con respecto a las prioridades sociales, la redefinición de los roles de los profesionales de la salud así como la demostración de su impacto sobre el estado de salud de las personas.

Para afrontar estos retos, 130 organizaciones y personas provenientes de todo el mundo -con responsabilidades en la educación de los profesionales sanitarios, en su regulación o en las políticas de salud- participaron durante ocho meses en un proceso Delphi de tres rondas que culminó en una conferencia de tres días para alcanzar un consenso.

El Consenso Global sobre la Responsabilidad Social de las Facultades de Medicina ${ }^{1}$ (GCSA) consistió en 10 directrices estratégicas dirigidas a las facultades de medicina a fin de que llegaran a ser socialmente responsables mediante las mejoras precisas para:

- Dar respuesta a las necesidades de la salud actuales y futuras y a las demandas de la sociedad.

- Reorientar sus prioridades educativas, de investigación y de asistencia según dichas necesidades.

- Reforzar su gobierno así como su asociación con otros agentes sociales interesados (stakeholders).

- Utilizar la evaluación y la acreditación basada en su desempeño (performance) y en los resultados obtenidos (outcomes).

\footnotetext{
1 El término social accountability es intraducible al castellano en su sentido estricto de 'rendición de cuentas'. En este texto se traduce por 'responsabilidad social', aun reconociendo la limitación que esto produce.
}

El consenso recomienda reforzar las sinergias entre las organizaciones y las redes existentes para progresar de forma consensuada hacia una acción global mediante una serie de actividades, tales como:

- Defensa y promoción del valor del consenso global.

- Consulta con el fin de la adaptación e implementación del consenso global en los diferentes contextos locales.

- Investigación para el diseño de los estándares que reflejen la responsabilidad social.

- Coordinación global para compartir experiencias y proporcionar apoyos.

Un siglo después del informe Flexner, el GCSA constituye un hito para el futuro de la educación médica en el mundo entero.

\section{Perspectiva}

Tras el informe Flexner sobre educación médica en Estados Unidos y Canadá, el principal desafío para la educación en las profesiones relacionadas con la salud en el siglo Xxi reside en la demostración, por parte de las instituciones educativas, de su contribución a la mejora del funcionamiento de los sistemas de salud y del estado de salud de la población, no sólo mediante la confección de programas educativos orientados hacia los problemas prioritarios de la salud, sino también mediante una mayor participación en la capacidad de anticiparse a las necesidades en el ámbito de la salud y los recursos humanos de un país, y en garantizar que sus titulados tengan trabajo donde más se los necesite para poder prestar los servicios demandados.

Se requiere un nuevo paradigma de excelencia para las instituciones académicas, así como un nuevo conjunto de estándares y mecanismos de acredi-
Versión castellana a cargo de Ángel Centeno y Ana Belén del Río, del Departamento de Educación Biomédica, Facultad de Ciencias Biomédicas, Universidad Austral, Argentina.

Versión original inglesa: www.healthsocialaccountability org

Para contactos:

gcsa@familymed.ubc.ca

(c) 2011 Educación Médica 
tación que promuevan y evalúen su capacidad para tener un mayor impacto en la salud.

Desde el 10 hasta el 13 de octubre de 2010, 65 delegados de instituciones de educación médica y de acreditación de todo el mundo se reunieron en East London (Sudáfrica) para finalizar la elaboración del GCSA, cuyos acuerdos se presentan a continuación. Ésta fue la culminación de un proceso de dos años con un grupo internacional de referencia (IRG) de 130 organizaciones y personas consideradas líderes en educación médica, acreditación y responsabilidad social.

Con la coordinación de un comité ejecutivo (SC) de 20 miembros, el IRG participó durante 8 meses en un proceso que incluyó tres etapas y que condujo al GCSA. Inicialmente se recogieron 43 páginas de datos que respondían a tres preguntas abiertas:

- ¿Cómo debería una facultad de medicina mejorar su capacidad para dar respuesta a los futuros desafíos sanitarios de la sociedad?

- ¿Cómo podría incrementarse esta capacidad, incluyendo el uso de sistemas de acreditación para la autoevaluación y la revisión por pares?

- ¿Cómo debería evaluarse el avance hacia este objetivo?

Tras dos rondas de trabajo y un encuentro, los temas se resumieron y se llegó a un consenso que se agrupó en 10 áreas temáticas. Cada área y su contenido provinieron entonces de un proceso generado a partir de las bases - lo que garantizó que el consenso se construyera fundamentado en la experiencia y los conocimientos de los miembros del IRGque se fue puliendo y negociando paulatinamente:

- Área 1. Previsión de las necesidades de salud de la sociedad.

- Área 2. Asociación con el sistema de salud y los grupos de interés.

- Área 3. Adaptación a los cambios de rol de los médicos y otros profesionales de la salud.

- Área 4. Fomento de la educación basada en resultados.

- Área 5. Creación de un gobierno de la facultad de medicina responsable y capaz de responder.

- Área 6. Redefinición del ámbito de incumbencia de los estándares educativos, de investigación y de asistencia.

- Área 7. Apoyo continuo para la mejora de la calidad en educación, investigación y asistencia.

- Área 8. Establecimiento de mecanismos obligatorios de acreditación.

- Área 9. Equilibrio entre los principios globales y la especificidad del contexto.

- Área 10. Definición del papel de la sociedad.
El propósito de esta iniciativa del GCSA fue obtener un acuerdo sobre el marco de trabajo necesario para que las facultades de medicina consigan un impacto mayor en el desempeño de los sistemas de salud y en el estado de salud de la población. En este marco de trabajo, esperamos poder establecer un conjunto de estándares de educación médica que reflejen esta capacidad y proponer métodos de evaluación, de acreditación y de mejora de la calidad.

Para poder concretar esta aspiración, el GCSA se concibió en tres fases:

- Fase I (febrero-octubre de 2010). Recopilación de opiniones de los miembros del IRG por medio del método Delphi. Cada consulta fue analizada por el SC y se devolvió a los miembros del IRG para una próxima ronda a fin de lograr un mayor consenso.

- Fase II (10-13 de octubre de 2010). Conferencia en East London, en la que participaron representantes de organizaciones interesadas en la mejora de la educación médica. El consenso al que se llegó en la conferencia se basó en el proceso Delphi de los meses previos.

- Fase III (tras la conferencia). Se establecerán colaboraciones, comités y nuevas iniciativas que ayudarán a llevar a la práctica las recomendaciones de la conferencia por medio de publicaciones, promoción y apoyo.

Actualmente estamos comenzando la fase III, que va a requerir de los esfuerzos concertados de un amplio grupo de personas y de iniciativas.

Junto con las diferentes instituciones representadas en el IRG, existe un gran mosaico de actores que trabaja colectivamente para lograr las mejoras deseadas.

El siguiente documento representa un consenso claro en esta dirección, para su implementación en 10 áreas interrelacionadas. Esto incluye el fortalecimiento y desarrollo de estándares de acreditación, sistemas y evaluación, todos ellos dedicados a la mejora de la calidad y su impacto en las necesidades de la salud de los ciudadanos en una escala local y global. Un avance en ese sentido será un legado valioso del siglo XXI.

El GCSA fue organizado por la University of British Columbia y la Walter Sisulu University, coincidiendo con la celebración del $25 .^{\circ}$ aniversario de la Walter Sisulu University School of Medicine, uno de los primeros ejemplos de una facultad de medicina socialmente responsable.

Agradecemos el apoyo de la Organización Mundial de la Salud, de la red de facultades de medicina 
TheNET, de la Sociedad Francófona de Educación Médica (SIFEM) y de la Federación Mundial de Educación Médica (WFME). El GCSA fue posible gracias al apoyo generoso mediante una subvención de Atlantic Philanthropies.

Estamos muy agradecidos por haber recibido el asesoramiento externo en el proceso de diseño y consenso de Louise O'Meara, del Instituto de Interacción para el Cambio Social.

\section{El documento de consenso}

El concepto de responsabilidad social abarca un amplio ámbito sistémico que va desde la identificación de las necesidades de la salud hasta la verificación de los efectos conseguidos sobre dichas necesidades. Las 10 áreas reflejan una secuencia lógica, a partir de la comprensión del contexto social, la identificación de los retos de las necesidades de la salud y el establecimiento de colaboraciones para poder actuar eficientemente (áreas 1 y 2). Además de la composición de los recursos profesionales precisos para afrontar las necesidades de la salud, se describen los roles y las competencias previstas para los médicos (área 3), como guía para las estrategias educativas (área 4), que las facultades de medicina deberán implementar junto con las estrategias correspondientes a la investigación y la prestación de servicios asistenciales (área 5). Es preciso establecer los estándares para que las instituciones intenten alcanzar niveles superiores de competencia (áreas 6 y 7) que las autoridades nacionales deben reconocer (área 8). Aunque la responsabilidad social es un valor universal (área 9), serán las sociedades locales las que, en última instancia, apreciarán los logros obtenidos (área 10).

\section{Área 1. Previsión de las necesidades de salud de la sociedad}

1.1 La facultad de medicina se guía en su desarrollo por valores básicos tales como relevancia, equidad, calidad, aplicación responsable de los recursos en cuanto a las necesidades, sustentabilidad, innovación y colaboración, los cuales también deben predominar en cualquier sistema de salud.

1.2 La facultad de medicina reconoce diversos factores sociales en torno a la salud (políticos, demográficos, epidemiológicos, culturales, económicos, ambientales) y dirige sus programas de enseñanza, investigación y asistencia de acuerdo con éstos.
1.3 La facultad de medicina tiene una visión y una misión tanto en educación (incluida la formación médica básica, de posgrado y continuada) como en investigación (incluidas la básica y la aplicada) y en asistencia que se basan principalmente en las necesidades actuales y futuras de la sociedad. La facultad de medicina prevé los cambios precisos para un sistema de salud eficiente y equitativo con recursos humanos competentes.

\section{Área 2. Asociación con el sistema de salud y los grupos de interés}

2.1 La facultad de medicina se compromete a trabajar en asociación cercana con los principales grupos interesados en la salud (por ejemplo, quienes establecen las políticas sanitarias, las organizaciones de atención de la salud, las asociaciones profesionales y otras profesiones de la sociedad) y en otros sectores para mejorar el funcionamiento de los sistemas de salud y elevar el estado de salud de la gente a través de su misión de docencia, investigación y asistencia.

2.2 La facultad de medicina se fortalece en su asociación, como lo evidencia la consulta continua y efectiva con los grupos mencionados anteriormente en el diseño, implementación y evaluación de sus programas de enseñanza, investigación y asistencia. Quienes colaboran mutuamente se apoyan unos a otros para cumplir su misión de atender las necesidades prioritarias de la salud y sus desafíos. La facultad de medicina asesora a las diversas autoridades respecto a las políticas y estrategias necesarias para lograr un sistema de salud aún más responsable socialmente.

2.3 La facultad de medicina reconoce que la comunidad local es la primera interesada y corresponsable de un conjunto de servicios de salud para una población determinada, en una región geográfica específica, que incluya aspectos de calidad, equidad, relevancia y eficiencia para el desarrollo y la evaluación de modelos de integración de la población y las actividades individuales, y para aprender y realizar investigación en salud.

2.4 La facultad de medicina reconoce que un sistema de salud sólido debe estar basado en un enfoque firme de atención primaria de la salud, con una correcta integración entre el primer nivel de atención y el segundo y tercer niveles, y con un equilibrio adecuado de disciplinas profesionales para satisfacer las necesidades de la 
población. Este enfoque debe verse reflejado en las facultades de medicina mediante programas de docencia, investigación y asistencia.

\section{Área 3. Adaptación a los cambios de rol de} los médicos y otros profesionales de la salud

3.1 La facultad de medicina brinda al titulado una serie de competencias que son coherentes con la evolución de las necesidades de la comunidad a la que sirve, con el sistema de salud en el que trabaja y con las expectativas de la sociedad. Las competencias se definen con la aprobación de las partes interesadas (incluidos otros profesionales de la salud y de otros sectores sociales) y teniendo en cuenta la imperativa necesidad de compartir y delegar eficientemente las tareas entre los miembros del equipo de salud, de modo que se garantice una asistencia accesible, eficiente y de calidad.

3.2 La facultad de medicina abarca un serie de competencias para el médico que se relacionan con los valores previamente descritos y con el concepto de profesionalismo tal como lo reconocen las organizaciones competentes. Estas competencias incluyen la ética, el trabajo en equipo, las aptitudes culturales, el liderazgo y la comunicación.

3.3 En consonancia con las necesidades que van apareciendo en la sociedad y con los ajustes del sistema de salud, la facultad de medicina produce una variedad de especialistas apropiada en cantidad y calidad. Se da atención prioritaria a los titulados comprometidos con la atención primaria.

3.4 La facultad de medicina reconoce que, independientemente de la especialidad que los futuros médicos elijan, éstos deben ser explícitamente activos en salud poblacional y su coordinación con la salud individual, en promoción de la salud y en prevención de riesgos y enfermedades, así como en la rehabilitación del paciente y de la comunidad en su totalidad. Los titulados son activos promotores de la salud y de las reformas del sistema de salud.

\section{Área 4. Fomento de la educación basada en resultados}

4.1 La facultad de medicina busca, selecciona y apoya a estudiantes que reflejen la diversidad social y los grupos desfavorecidos.

4.2 Todo el espectro de intervenciones educativas (incluyendo el contenido y la estructura curricular, la asignación de recursos, los métodos de enseñanza, el desarrollo docente y los sistemas de evaluación) deben organizarse de acuerdo con las necesidades individuales y sociales.

4.3 Las oportunidades de aprendizaje y las instalaciones están generalmente disponibles para ayudar a quienes desean adquirir habilidades de aprendizaje continuo y competencias tales como solución de problemas y promoción, que serán necesarias para preparar a los titulados para futuros roles de liderazgo.

4.4 A los estudiantes se les ofrece un contacto temprano y longitudinal con experiencias de aprendizaje en la comunidad, tanto en la teoría como en la práctica, para entender y actuar sobre los condicionantes de la salud y adquirir habilidades clínicas apropiadas. Este tipo de entrenamiento está integrado en todas las disciplinas con un compromiso global de los docentes y un uso coherente de los recursos en beneficio de la comunidad.

4.5 La facultad de medicina ofrece un abanico de servicios y mecanismos de apoyo a sus docentes y estudiantes para implementar estrategias educativas y asegurarse de que sus titulados poseen las competencias esperables requeridas en un sistema socialmente responsable.

4.6 La facultad de medicina evalúa regularmente el desempeño de los estudiantes en relación con la adquisición de las competencias tal como se describe en el área 3.

4.7 La estrategia y los métodos educativos se revisan y se actualizan periódicamente de acuerdo con prácticas de buena educación médica, evaluación del aprendizaje de los estudiantes, las experiencias de los titulados en la práctica médica y según el feedback de los estudiantes y de las partes interesadas del sistema de salud. Esta revisión incluye una atención explícita a la coherencia entre los valores institucionales proclamados y las políticas y prácticas observadas.

\section{Área 5. Creación de un gobierno de la facultad de medicina responsable y capaz de responder}

5.1 La facultad de medicina debe generar un gobierno y un liderazgo responsable para expresar su papel como un actor clave en el desarrollo del sistema de salud y la fuerza laboral, mediante la integración de principios de responsabilidad social y programas de docencia, investigación y asistencia.

5.2 La facultad de medicina cuenta con el compromiso de todo su equipo académico y de los estudiantes para hacer frente a los desafíos de la salud y las necesidades de la sociedad. Este com- 
promiso se reconoce y se valora críticamente por medio de la verificación regular y sistemática con instrumentos certificados.

5.3 La facultad de medicina desarrolla la cooperación con otros actores interesados (incluyendo otras facultades de ciencias de la salud) para optimizar su funcionamiento y para lograr la cantidad y calidad de titulados deseada, con el fin de alcanzar un impacto en la salud.

5.4 La facultad de medicina se asegura de que los recursos existentes se asignen apropiadamente y se administren eficientemente, e intenta que los nuevos recursos le permitan funcionar como una institución socialmente responsable. La facultad de medicina se compromete a que los recursos garanticen un número adecuado de docentes cualificados, una infraestructura que funcione correctamente y que se implementen nuevos programas, siempre teniendo en cuenta un equilibrio apropiado entre todos los niveles de prestación de servicios sanitarios.

\section{Área 6. Redefinición del ámbito de incumbencia de los estándares educativos, de investigación y de asistencia}

6.1 La excelencia académica se entiende como la capacidad de ofrecer programas de educación, investigación y asistencia que respondan de la mejor manera a los desafíos de la salud y las necesidades de la sociedad y que produzcan un impacto positivo la salud. En consonancia con los principios de responsabilidad social, la consecución de los estándares educativos refleja una constante en lo que se refiere a identificación de problemas, elecciones estratégicas, procesos de administración, logros e impacto en la salud, tanto individual como poblacional.

6.2 Los estándares existentes en educación médica se revisan y se enriquecen con nuevos estándares, de manera que su alcance abarque la obtención de información (quién se forma y dónde), procesos, logros (aquello que los titulados hacen efectivamente en la práctica) e impacto. Los estándares reflejan la continuidad desde el pregrado hasta el posgrado, incluyendo el desarrollo profesional continuo. Los estándares en programas de investigación y asistencia se orientan también a considerar necesidades definidas, y la satisfacción de esas necesidades se evalúa y se brinda como retroalimentación a los responsables.

6.3 Los estándares relacionados con programas de educación cubren: articulación de las competencias esperables; coordinación con otros profe- sionales de la salud; diseño y renovación del currículo; coordinación y apoyo para su puesta en práctica; asignación de recursos y administración, evaluación de estudiantes, programas y docentes; verificación de la adquisición de competencias por todos los titulados; evaluación permanente de la elección de especialidades y compromiso profesional de los titulados de dar asistencia en aquellos lugares de mayor necesidad. Estos estándares se articulan y se gestionan de manera que apoyen cambios innovadores e incrementen la creatividad para responder a los requerimientos de la sociedad.

6.4 Los estándares relacionados con los logros y el impacto educativo cubren: elección por parte de los titulados de carreras relevantes en cuanto a los desafíos y necesidades, implementación y retención de titulados donde más se los necesite, capacidad de los titulados para encarar apropiadamente aspectos de la salud, ambiente laboral propicio para los titulados, contribución a la mejora del estado de salud de la población general donde la facultad de medicina se halle.

6.5 Los estándares relacionados con el gobierno de la facultad de medicina cubren: calidad en el gobierno institucional, liderazgo correcto, profesionalismo de los docentes, uso apropiado de los recursos y capacidad para generar y mantener contactos sólidos con otros grupos interesados en los sistemas de salud. Todos ellos contribuyen a la aplicación de los principios de responsabilidad social en la práctica.

6.6 Los estándares de acreditación abarcan experiencias de educación interprofesional y aseguran las capacidades requeridas por los titulados para aprender con, sobre, para y de otros profesionales.

Área 7. Apoyo continuo para la mejora de la calidad en educación, investigación y asistencia

7.1 La facultad de medicina se compromete a un proceso periódico e interno de revisión y mejora de la calidad guiado por estándares definidos a través de la educación, la investigación y la asistencia. El cumplimiento de estos estándares es una parte esencial de una facultad de medicina socialmente responsable.

7.2 La facultad de medicina evalúa su progreso hacia la responsabilidad social a través de una serie de medidas, tanto cualitativas como cuantitativas, que reflejan su desempeño de acuerdo con un aporte válido y confiable y según estándares de acreditación de procesos y de logros. 
7.3 La facultad de medicina apoya completamente el uso de instrumentos de medición, y los utiliza sistemática y periódicamente para la mejora de la evaluación institucional. El proceso es explícito, transparente, constructivo y abierto a otros grupos interesados.

7.4 La facultad de medicina reconoce que una estructura de gobierno capaz, el liderazgo responsable y la determinación de estándares profesionales para la educación médica, la investigación y la asistencia, así como los docentes e integrantes, son determinantes para la mejora de la calidad y el progreso hacia la transformación en una facultad de medicina socialmente responsable.

\section{Área 8. Establecimiento de mecanismos obligatorios de acreditación}

8.1 La acreditación es una poderosa fuerza para el cambio institucional y su mejora, y debe ser apoyada activamente por las autoridades académicas y nacionales de la salud en el mundo entero. Todas las facultades de medicina deben estar acreditadas por una agencia reconocida según los mecanismos establecidos en cada país o región. La acreditación debe realizarse a intervalos regulares, y entre estos intervalos se deben llevar a cabo mejoras.

8.2 Los estándares y procesos de acreditación reflejan claramente los principios de responsabilidad social, al tiempo que incorporan los aportes, procesos, logros e impacto para evaluar y promover la capacidad de las facultades de medicina de responder con eficiencia a los desafíos de salud y las necesidades de la sociedad.

8.3 La existencia de mecanismos de acreditación implica la presencia de apoyo al esfuerzo de las facultades de medicina para cumplir con los estándares y procesos arriba mencionados. Dependiendo de cada contexto, este apoyo puede ser tan variado como la formulación de directrices de corte político que aumenten la responsabilidad social y la prestación de recursos e incentivos.

8.4 La evaluación interna se refuerza con la revisión externa por pares. Representantes de los grupos interesados han de involucrarse en la definición de estándares de evaluación, en la selección de revisores externos y en la revisión periódica del sistema de acreditación.

\section{Área 9. Equilibrio entre los principios globales y la especificidad del contexto}

9.1 Los principios de responsabilidad social son uni- versales: se deben adoptar y aplicar en todo el mundo. Asimismo, incrementan la capacidad de las facultades de medicina para usar de la mejor manera su potencial para identificar y afrontar los desafíos de la salud y las necesidades de la sociedad con un espíritu de calidad, equidad, relevancia, innovación y de uso apropiado de los recursos.

9.2 Como consecuencia del incremento de movilidad de los médicos y de los pacientes, la facultad de medicina debe incorporar una dimensión internacional. A fin de contribuir a un desarrollo global sostenible, las facultades de medicina deben apuntar hacia la integración de perspectivas globales e interculturales al definir sus propósitos y su organización, así como al ejecutar sus programas educativos.

9.3 Mientras que los principios, definiciones y clasificaciones de las facultades de medicina socialmente responsables pueden tener relevancia global, su adaptación a cada contexto local es esencial.

9.4 Las organizaciones internacionales de salud y educación superior (regionales o globales) deben abogar por los sistemas que aseguren la calidad, incluyendo la acreditación y los marcos regulatorios, para aplicar los principios de responsabilidad social y hacerse cargo de las acuciantes necesidades de salud de los países mientras hacen frente a la crisis general en el desarrollo de los recursos humanos en la salud.

\section{Área 10. Definición del papel de la sociedad}

10.1 Debe lograrse un equilibrio entre la preservación de la autonomía institucional y el papel de los grupos de interés y de la sociedad civil en la incorporación de la responsabilidad social en las facultades de medicina. Éste es un verdadero reto.

10.2 Los principales interesados - por ejemplo, quienes hacen las políticas de salud, los gestores de los servicios de salud, los profesionales de la salud y la sociedad civil- deben estar representados en equipos de evaluación internos y externos, incluidos para la acreditación, ya que es deseable rendir cuentas a aquellos a los que se intenta servir o con quienes se trabaja. Los representantes de los grupos de interés deben tener un compromiso explícito hacia los valores fundamentales que se tienen en común y hacia los principios de responsabilidad social.

10.3 Las comunidades en las que se ubican las facultades de medicina deben presentar sus ob- 
servaciones con frecuencia respecto al nivel de responsabilidad social de la facultad. Estas observaciones, en cuanto al estado de la acreditación de la facultad, deben estar a disposición de la comunidad.

\section{Comité ejecutivo}

Magdalena Awases

Human Resources for Health (HRH), WHO Regional Office for Africa HRH, WHO Geneva

Rebecca Bailey a Charles Boelen ${ }^{a}$ Mario dal Poz Moses Galukande ${ }^{\text {a }}$ ex-HRH, WHO HRH, WHO Geneva Makerere University and International Medical Group

Jorge E. Gutiérrez Calivá Asociación Costarricense de Facultades y Escuelas de Medicina (ACOFEMED)

Dan Hunt Liaison Committee on Medical Education (LCME)

Jehu Iputo ${ }^{\mathrm{a}}$

Yusuf Irawan

Ahmed Kafajei

Joël Ladner ${ }^{\mathrm{a}}$

Sam Leinster ${ }^{a}$

Stefan Lindgren ${ }^{a}$

Khaya Mfenyana ${ }^{a}$

Tewfik Nawar ${ }^{\text {a }}$

André-Jacques Neusy ${ }^{\mathrm{a}}$

Walter Sisulu University

Hasanuddin University

Jordan University of Science and

Technology

Université de Rouen

University of East Anglia

World Federation of Medical Education (WFME) and Lund University

Walter Sisulu University

Université de Sherbrooke

Training for Health Equity Network (THEnet)

Björg Pálsdóttir ${ }^{a}$

THEnet

Jean Rochon

Université Laval

University of British Columbia

Copresidentes

Charles Boelen: boelen.charles@wanadoo.fr

Robert Woollard:woollard@familymed.ubc.ca

\section{Grupo internacional de referencia}

Mohamed Elhassan

Abdalla ${ }^{a}$

Ibrahim Abdulmeini

Walid Abubaker ${ }^{a}$

Liliana Arias

Carol Aschenbrener

Makonnen Asefa ${ }^{a}$

Sébastien Audette

Djona Avoksouma

Jazan University

Registrars of the Medical \& Dental Council of Nigeria

WHO/EMRO

Asociación Colombiana de Facultades de Medicina. Miembro del Comité de Administración de FEPAFEM

Association of American Medical Colleges (AAMC)

Ethiopian Public Health Association/World Federation of Public Health Associations (WFPHA)

Accreditation Canada

WHO Africa Office
Ibrahim Bani-Hani

Barbara Barzansky

Mourad Belaciano

Dan Benor

John Bligh

Daniel Blumenthal ${ }^{\mathrm{a}}$

Bettina Borisch

Rosa M. Borrell-Bentz

Nick Busing

David Buso ${ }^{a}$

Jim Campbell

Emmanuel Cassimatis

Francesca Celletti

Ángel Centeno ${ }^{\mathrm{a}}$

Lincoln Chen

Shakuntala Chhabra ${ }^{a}$

Ian Couper ${ }^{\mathrm{a}}$

Manuel Dayrit

Milton de Arruda Martins

Eric de Roodenbeke

Jean-François Denef ${ }^{a}$

Horacio Deza

Mohenou Isidore

Jean-Marie Diomande

Robbert Duvivier

Tetanye Ekoe ${ }^{\text {a }}$

Amal Elouazzani ${ }^{\text {a }}$

Mohamed Elsanousi ${ }^{\text {a }}$

Julian Fisher

Antoine Flahault
Fortunato Cristobal

Jean-Paul Francke

Tabeh Freeman

Seble Frehywot

George Gage

Jacob Gayle

Grace George ${ }^{a}$

Trevor Gibbs

Charles Godue

Ioana Goganau a
Association of Medical Education in the Eastern Mediterranean Region

Supreme Council of Egyptian Universities

LCME

Asociación Brasileña de Educación Médica (ABEM)

Ben-Gurion University of Negev Academy of Medical Educators Morehouse School of Medicine WFPHA

Human Resources Development, WHO Regional Offices for the Americas/PAHO

Association of Faculties of Medicine of Canada (AFMC)

Walter Sisulu University

Educational Commission for Foreign Medical Graduates (ECFMG) HRH, WHO Geneva

Universidad Austral

China Medical Board

Mahatma Gandhi Institute of Medical Science (MGIMS)

University of the Witwatersrand HRH, WHO Geneva

University of Sao Paulo International Hospital Federation (IHF-FIH)

International French Speaking Society for Medical Education (SIFEM)

Asociación de Facultades de Ciencias Médicas de la República Argentina (AFACIMERA) UFR-Sciences Médicales d'Abidjan

International Federation of Medical Students' Associations (IFIMSA) Yaounde Faculty of Medicine Hassan II University

FDI World Dental Federation Association of Schools of Public Health in the European Region (ASPHER)

Ateneo de Zamboanga University International Association of Deans of Francophone Medical Schools (CIDMEF)

Liberia Medical School

George Washington University

Afro-European Medical and Research Network

Ford Foundation

Walter Sisulu University

AMEE

Human Resources Development, WHO Regional Offices for the Americas/PAHO

IFMSA 


\section{Rajesh Gongal ${ }^{\text {a }}$ \\ David Gordon a \\ Arcadi Gual \\ Edward Gyader ${ }^{\text {a }}$ \\ Georges Haddad \\ John Hamilton a \\ Bashir Hamad ${ }^{\text {a }}$ \\ Jason Hilliard \\ Yiqun $\mathrm{Hu}$ \\ Manuel Huamán \\ Muzaherul Huq \\ Yojiro Ishii}

Marian Eslie Jacobs ${ }^{\mathrm{a}}$

Susan Johnson a

Samuel Kargbo

Geoffrey Kasembeli

Zulfiqar Khan ${ }^{\text {a }}$

Michael Kidd

Khunying Kobchitt

Limpaphayom ${ }^{\text {a }}$

Joseph Kolars

Jan de Maeseneer

Henri Manasse a

Dianne Manning a

Maurice McGregor ${ }^{\text {a }}$

Jim McKillop

Donald Melnick

Hugo Mercer

Mwapatsa Mipando ${ }^{a}$

Nader Momtazmanesh ${ }^{a}$

Gottlieb Monekosso ${ }^{\text {a }}$

Fitzhugh Mullan

Richard Murray a

Jeremiah Mwangi

Rose Chalo Nabirye a Sophon Napathorn ${ }^{a}$ Lois Nora ${ }^{a}$
Patan Academy of Health Sciences, Patan Hospital

Association of Medical Schools in Europe (AMSE)

Fundación Educación Médica

School of Medicine and Health

Sciences. University of Ghana

UNESCO

University of Newcastle

University of Gezira

University of Colorado

Shanghai Jiao Tong University

Asociación Peruana de Facultades

de Medicina (ASPEFAM)

$\mathrm{HRH}$, WHO Regional Office for

South-East Asia

Japan International Cooperation Agency

Association of Medical Schools in Africa (AMSA) \& University of Cape Town

National Board of Medical Examiners (NBME) \& University of Iowa

Ministry of Health, Sierra Leone

Afro-European Medical and Research Network

WHO Pakistan

Wonca Working Party on Education South-East Asian Regional Association on Medical Education Bill \& Melinda Gates Foundation The Network Towards Unity for Health (TUFH)

American Society of Health-System Pharmacists \& International Pharmaceutical Federation (IPF) University of Witwatersrand

McGill University

General Medical Council (GMC)

NBME

Former HRH, WHO

University of Malawi

Ministry of Health \& Medical Education

Global Health Dialogue

George Washington University Australia International Alliance of Patients' Organizations

WFPHA, Uganda

Chulalongkorn University

North-Eastern Ohio Universities \& Colleges of Medicine and Pharmacy \& ICME
John Norcini ${ }^{\text {a }}$

Ezekiel Nukuro

Chacha Nyaigotti-Chacha Inter-University Council for East

Francis Omaswa

Alberto Oriol Bosch

Martins Ovberedjo a

Neil Pakenham-Walsh

Jordi Palés

Madalena Patricio ${ }^{a}$

Galina Perfilieva

Dominique Pestiaux

David Prideaux ${ }^{a}$

Pablo Pulido

Rich Roberts

Paschalis Rugarabam ${ }^{a}$

Mubashar Sheikh

Jusie Siega-Sur a

Lesley Southgate

David Stern

Roger Strasser

Navin Sunderlall ${ }^{a}$

Kate Tulenko

Felix Vartanian ${ }^{\mathrm{a}}$

Anvar Velji a

Kuku Voyi

Margot Weggemans

Gustaaf Wolvaardt

Liz Wolvaardt ${ }^{\text {a }}$

Paul Worley

Akemi Yonemura

Toshimasa Yoshioka Africa (IUCEA)

Foundation for Advancement of International Medical Education and Research (FAIMER)

Asociación Mexicana de Facultades de Medicina (AMFEM) $\mathrm{HRH} / \mathrm{WHO}$ Regional Office for the Western Pacific

African Center for Global Health and Social Transformation Fundación Educación Médica WHO Tanzania

Global Healthcare Information Network

Sociedad Española de Educación Médica (SEDEM)

AMEE

WHO Europe Office

SIFEM

Flinders University

Panamerican Federation of Associations of Medical Schools (PAFAMS/FEPAFEM)

Wonca President elect

Hubert Kairuki Memorial University

Global Health Workforce Alliance (GHWA)

University of the Philippines

Academy of Medical Educators

Institute of International Medical Education

Northern Ontario School of Medicine

University of Kwazulu Natal

IntraHealth International

Russian Academy of Advanced Medical Studies

Global Health Education Consortium

University of Pretoria

IFMSA

Foundation for Professional Development

University of Pretoria

Flinders University

UNESCO

Association of Medical Schools in the Western Pacific Region

\footnotetext{
${ }^{a}$ Asistentes a la conferencia GCSA celebrada en East London, Sudáfrica, del 10 al 13 de octubre de 2010.
} 